\title{
Prospects of Perineural Administration of Autologous Mesenchymal Stem Cells of Adipose Tissue in Patients with Cerebral Infarction
}

\author{
Shanko Yuri ${ }^{1}$, Navitskaya Valeria ${ }^{1}$, Zamaro Alexandra ${ }^{2}$, Krivenko Svetlana ${ }^{3}$, Zafranskaya Marina ${ }^{4}$, Pashkevich \\ Svetlana $^{2}$, Koulchitsky Stanislav ${ }^{5}$, Takalchik Stukach Yulya ${ }^{2}$, Denisov Andrew ${ }^{2}$ and Kulchitsky Vladimir*2 \\ ${ }^{1}$ Republican Scientific and Practical Center of Neurology and Neurosurgery, Minsk, Belarus \\ ${ }^{2}$ Institute of Physiology, National Academy of Sciences, Minsk, Belarus \\ ${ }^{3}$ Minsk Scientific-Practical Center for Surgery, Transplantation and Hematology, Minsk, Belarus
}

${ }^{4}$ Belarusian Medical Academy of Post-Graduate Education, Minsk, Belarus

${ }^{5}$ Liege University, Liege, Belgium

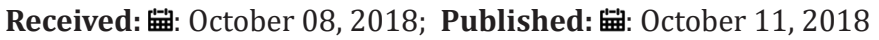

*Corresponding author: Kulchitsky Vladimir, Institute of Physiology, National Academy of Sciences, Minsk, Belarus, Europe

\section{Abstract}

Background: Frustrating statistics of therapy of patients with cerebral infarctions is well known. Longstanding deficiency of somatic and visceral functions is one of the negative consequences of existing therapy. Such state is developed due to persistent structural and functional lack of central control of various body activities by brain neural networks. Finally, most of the patients have severe invalidities even in distant period after performed treatment. Therefore, development and implementation of technologies that will allow activating reparative processes in brain after cerebral infarction is one of prospective tasks.

Aims: development and implementation of new technique of cerebral infarctions treatment using autologous mesenchymal stem cells (MSC) of adipose tissue.

Methods: Authors developed unique technique aimed at formation of conditions for natural migration of stem cells (SC) to the area of cerebral infarction after their endoscopic administration into nasal submucosa.

Results: Autologous mesenchymal stem cells (MSC) of 25 patients naturally migrated to the area of cerebral infarction after their administration into nasal submucosa. The process of MSC differentiation into neuron-like elements after penetration to cranial cavity was previously shown in experiments. Patients with primary cerebral infarctions $(n=12)$ and secondary ones $(n=8)$ showed statistically significant improvement of physiological functions control in 6 months after course therapy with SC assessed by National Institutes of Health Stroke Scale (NIHSS) and Glasgow Coma Scale (GCS), respectively. Administration of allogeneic SC to patients with cerebral infarctions $(n=5)$ was ineffective.

Discussion and Conclusion: Combination of standard therapy of cerebral infarctions with endoscopic perineural implantation of autologous MSC of adipose tissue is accompanied with activation of reparative processes leading to recovery of neurologic functions.

Keywords: Cerebral infarction, Mesenchymal stem cells, Perineural implantation, Patient, Reparative process

Abbreviations: GCS: Glasgow Coma Scale; MSC: Mesenchymal Stem Cells; NIHSS: National Institutes of Health Stroke Scale; SC: Stem Cells

\section{Introduction}

The search for "brain infarction mesenchymal stem cell therapy" in PubMed on October 01, 2018 showed 233 results. Experience with MSC therapy in patients with cerebral infarction is described in 34 articles and search for word combination "brain infarction mesenchymal stem cell autologous therapy patient" showed only 9 links. Authors want to pay attention that there are no results of search for "brain infarction mesenchymal stem cell autologous perineural therapy patient". Therefore, there is an empty informational space that should be filled with definite information. Authors recently had only experimental skills in MSC perineural implantation [1-3]. There are several studies in experimental and clinical practice when relatively new techniques of SC use are tested $[3,4]$. SC are accumulated around damaged brain regions after their local implantation through trepanation hole to the area of brain infarct and after systemic injection into bloodstream. Then 
SC differentiate into neuron-like elements [5]. It is obvious that technique of MSC perineural implantation into peripheral areas of cranial nerve endings (e.g. nasal cavity) [1-3] positively differs from other cellular technologies such as intracerebral MSC implantation [6], MSC implantation into cerebrospinal fluid [7] or bloodstream [5-7].

\section{Materials and Methods}

Technique of perineural delivery of MSC into brain after their endoscopic administration into nasal submucosa was developed and implemented $[1,3]$. The method was used in addition to standard therapy of acute cerebral infarction. 25 patients were examined. 12 patients with primary cerebral infarctions were included into first group, 8 patients with secondary brain infarctions after intracranial hemorrhages - into second group (aged 27-73 years, 40.25 mean). Patients of first and second groups were subjected to intranasal perineural administration of MSC using the previously described technique [3]. Patients with primary cerebral infarctions from the third group $(n=5)$ received standard therapy with allogeneic MSC. $50 \mathrm{ml}$ of adipose tissue was taken from umbilical area of each patient from the first and second groups. Cells were cultivated using previously described technique [3] and then endoscopic threefold intranasal implantation of autologous MSC in the amount of 5-12×106 cells was performed with the intervals of 5-9 days. Allogeneic SC were prepared from donor material in the absence of conditions for autologous tissues retrieval and administered perineurally to patients from the third group.

\section{Results}

Use of allogeneic MSC by means of their perineural delivery to brain resulted in improvement of functional state in no one of five patients from the third group during first days of observation. The state of patients from the first and second groups progressively improved after combination of classic therapy of cerebral infarctions with intranasal implantation of autologous MSC. This outcome is a valid argument in discussion on reasonability of autologous and allogeneic biomaterial use. Obtained data allow recommending autologous MSC as basic cellular technology for therapy of cerebral infarction. This point of view is confirmed by next data: all the patients from the first group showed stable (within 1 point of British medical research council scale) recovery of certain neurologic functions in 24 hours after each implantation of autologous MSC (Figure 1).

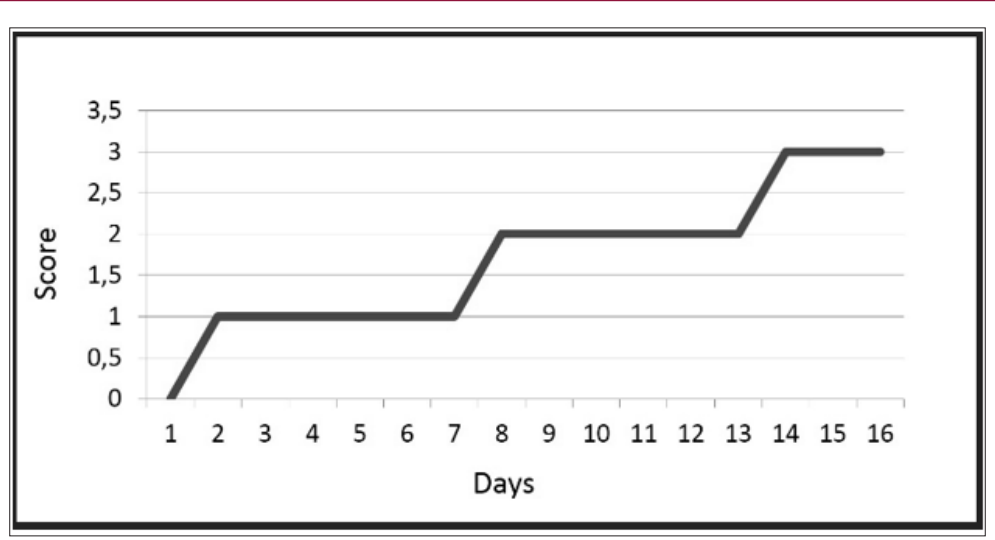

Figure 1: Efficacy assessment of cell therapy of primary cerebral infarctions $(n=12)$ was performed in the early period after implantation and in 6 months. Injections were made on Day 1 (first), Day 7 (second) and Day 13 (third).

In particular, NIHSS score changed in patients with primary cerebral infarctions $(n=12)$ from 14.2 at the beginning to 1.8 in 6 months; none of them had recurrent cerebral infarctions during one year of observation. In comparison, NIHSS score changed in patients of control group ( $n=20$, classic therapy of cerebral infarctions) from 14.4 at the beginning to 9.8 in 6 months; three of them (15\%) had recurrent cerebral infarctions during six months of observation.

Neurologic deficiency was assessed using GCS in patients with multiple secondary cerebral infarctions $(n=8)$ because all of them were in comatose state and NIHSS assessment was not applicable. Combined therapy (classic standard of care and administration of autologous MSC) was started at 6.2 GCS. Second group of patients did not show rapid regress of neurologic disorders, even after each SC implantation. These patients had GCS score 9.2 after the end of therapy with SC and NIHSS score 12.3 in six months after perineural implantation of MSC and standard therapy together with rehabilitation. It was hard to compare with control group, because one patient died there, three were in vegetative state and the last one had GCS score 28.0. However, it is important that there were no complications in any case.

\section{Discussion and Conclusion}

Combination of classic therapy of cerebral infarctions with endoscopic intranasal perineural implantation of autologous MSC, which migrate to the area of brain infarct, is accompanied with formation of conditions for activation of reparative processes leading to recovery of neurologic functions $[3,8]$.

Positive results of clinical observations also led to appearance of several conclusions and questions. No clear dependence was established between effectiveness of reparative processes and amount of implanted SC [8,9]. Diversity of effects determined by 
individual features of patients and variety of clinical performance of cerebral infarction was revealed. No dependence between reparative effect and the period of cellular therapy beginning was established. Relatively short period of observation (about one year) did not give definite answer to the question on possibility of long-term effect of cellular therapy (e.g. in one-two years) after cerebral infarction development and beginning of therapy. There are also some questions remained unclear according effectiveness of additional implantations of autologous MSC of adipose tissue in distant periods after treatment beginning (after one or more years). The mechanism of neurotrophic effects after autologous MSC use and recovery of neural network functions in brain was supposed, but not proved $[3,10,11]$. Therefore, the future task aimed at analysis of activation of neurotrophic and other endogenous reparative processes after SC implantation remains significant $[1,3,12,13]$.

\section{Acknowledgement}

This pooled analysis was funded by SSTP "New methods of medical care", section "Transplantation of cells, tissues and organs" (2016-2020), and by grant 000 "Synergy".

\section{References}

1. Kulchitsky V, Zamaro A, Shanko Y, Koulchitsky S (2018) Positive and negative aspects of cell technologies in cerebral diseases. J Neurol Stroke 8(2): 87-88.

2. Kulchitsky V, Zamaro A, Pashkevich S, Sushko T, Koulchitsky S (2018) Nasolacrimal way of cells implantation. J Neurol Stroke 8(2): 110-111.

3. Shanko Y, Navitskaya V, Zamaro A, Zafranskaya M, Krivenko S, et al. (2018) Somatotopic principle of perineural implantation of stem cells in patients with brain injuries. J Neurol Stroke 8(5): 259-261.

ISSN: 2574-1241

DOI: 10.26717/BJSTR.2018.10.001884

Kulchitsky Vladimir. Biomed J Sci \& Tech Res

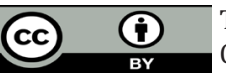

This work is licensed under Creative Commons Attribution 4.0 License

Submission Link: https://biomedres.us/submit-manuscript.php
4. Oppliger B, Joerger-Messerli M, Mueller M, Reinhart U, Schneider P, et al. (2016) Intranasal Delivery of Umbilical Cord-Derived Mesenchymal Stem Cells Preserves Myelination in Perinatal Brain Damage. Stem Cells Dev 25(16): 1234-1242.

5. Dalous J, Pansiot J, Pham H, Chatel P, Nadaradja C, et al. (2013) Use of human umbilical cord blood mononuclear cells to prevent perinatal brain injury: A preclinical study. Stem Cells Dev 22(1): 169-179.

6. Muir KW (2017) Clinical trial design for stem cell therapies in stroke: What have we learned? Neurochem Int 106:108-113.

7. Mazzini L, Mareschi K, Ferrero I, Vassallo E, Oliveri G, et al. (2006) Autologous mesenchymal stem cells: Clinical applications in amyotrophic lateral sclerosis. Neurol Res 28(5): 523-526.

8. Kalladka D, Sinden J, Pollock K, Haig C, McLean J, et al. (2016) Human neural stem cells in patients with chronic ischaemic stroke (PISCES): A phase 1, first-in-man study. Lancet 388(10046): 787-796.

9. Rossetti T, Nicholls F, Modo M (2016) Intracerebral Cell Implantation: Preparation and Characterization of Cell Suspensions. Cell Transplant 25(4): 645-664.

10. Nakamura M, Samii A, Lang JM, Götz F, Samii M, et al. (2016) De Novo Arteriovenous Malformation Growth Secondary to Implantation of Genetically Modified Allogeneic Mesenchymal Stem Cells in the Brain. Neurosurgery 78(4): E596-600.

11. Carvajal HG, Suárez-Meade P, Borlongan CV (2016) Amnion-derived stem cell transplantation: A novel treatment for neurological disorders. Brain Circ 2(1): 1-7.

12. Kokaia Z, Darsalia V (2018) Human Neural Stem Cells for Ischemic Stroke Treatment. Results Probl Cell Differ 66: 249-263.

13. Moritz CT (2018) Now is the Critical Time for Engineered Neuroplasticity. Neurotherapeutics 15(3): 628-634.

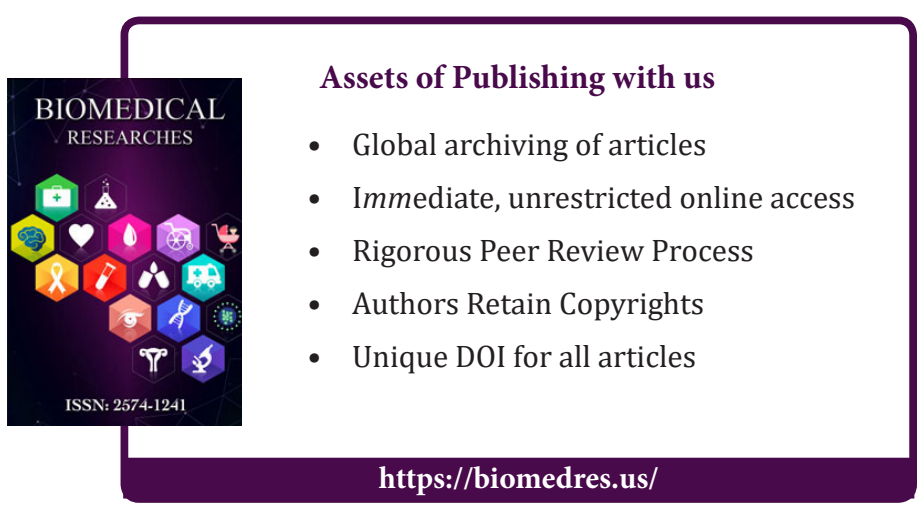

Cite this article: Shanko Y, Navitskaya V, Zamaro A, Krivenko S, Zafranskaya M, Pashkevich S, Koulchitsky S, Takalchik Stukach Y, Denisov A, Kulchitsky V. Prospects of Perineural Administration of Autologous Mesenchymal Stem Cells of Adipose Tissue in Patients with Cerebral Infarction. Biomed J Sci\&Tech Res 10(1)-2018. BJSTR. MS.ID.001884. DOI: 10.26717/ BJSTR.2018.10.001884. 